\title{
Interpretation of LHCb Hidden-Charm Pentaquarks within the Compact Diquark Model
}

\author{
Ahmed Ali, ${ }^{a}$ Ishtiaq Ahmed, ${ }^{b}$ M. Jamil Aslam, ${ }^{c}$ Alexander Parkhomenko ${ }^{d, *}$ and \\ Abdur Rehman ${ }^{b}$ \\ ${ }^{a}$ Deutsches Elektronen-Synchrotron DESY, \\ D-22607 Hamburg, Germany \\ ${ }^{b}$ National Centre for Physics, Quaid-i-Azam University Campus, \\ Islamabad 45320, Pakistan \\ ${ }^{c}$ Physics Department, Quaid-i-Azam University, \\ Islamabad 45320, Pakistan \\ ${ }^{d}$ Department of Theoretical Physics, P. G. Demidov Yaroslavl State University, \\ Sovietskaya 14, 150003 Yaroslavl, Russia \\ E-mail: ahmed.ali@desy.de, ishtiaqmusab@gmail.com, \\ muhammadjamil.aslam@gmail.com, parkh@uniyar.ac.ru, \\ Abdur.Rehman@fuw.edu.pl
}

The LHCb collaboration have recently updated their analysis of the resonant $J / \psi p$ mass spectrum in the decay $\Lambda_{b}^{0} \rightarrow J / \psi p K^{-}$, making use of their combined Run 1 and Run 2 data. In the updated analysis, three narrow states, $P_{c}(4312)^{+}, P_{c}(4440)^{+}$, and $P_{c}(4457)^{+}$, are observed. The spin-parity assignments of these states are not yet known. We interpret these narrow resonances as compact hidden-charm diquark-diquark-antiquark pentaquarks. Using an effective Hamiltonian, based on constituent quarks and diquarks, we calculate the pentaquark mass spectrum for the complete $S U(3)_{F}$ lowest $S$ - and $P$-wave multiplets, taking into account dominant spin-spin, spinorbit, orbital and tensor interactions. The resulting spectrum is very rich and we work out the quark flavor compositions, masses, and $J^{P}$ quantum numbers of the pentaquarks. However, heavy quark symmetry restricts the observable states in $\Lambda_{b}$-baryon, as well as in the decays of the other weakly-decaying $b$-baryons, $\Xi_{b}$ and $\Omega_{b}$. In addition, some of the pentaquark states are estimated to lie below the $J / \psi p$ threshold in $\Lambda_{b}$-decays (and corresponding thresholds in $\Xi_{b^{-}}$and $\Omega_{b^{-}}$ decays). They decay via $c \bar{c}$ annihilation into light hadrons or a dilepton pair, and are expected to be narrower than the $P_{c}$-states observed. We anticipate their discovery, as well as of the other pentaquark states present in the spectrum at the LHC, and in the long-term future at a Tera- $Z$ factory.

40th International Conference on High Energy physics - ICHEP2020

July 28 - August 6, 2020

Prague, Czech Republic (virtual meeting)

${ }^{*}$ Speaker 
Recently, the LHCb collaboration have presented an updated account of the resonant $J / \psi p$ mass spectrum in the decay $\Lambda_{b}^{0} \rightarrow J / \psi p K^{-}$, based on the combined Run 1 and Run 2 data, adding up to $9 \mathrm{fb}^{-1}$ [1]. In this analysis, which supersedes their earlier findings from 2015 [2], nominal fits of the data have been performed with an incoherent sum of Breit-Wigner amplitudes, which have resulted in the observation of three peaks $P_{c}(4312)^{+}, P_{c}(4440)^{+}$, and $P_{c}(4457)^{+}$with masses $4311.9 \pm 0.7_{-0.6}^{+6.8} \mathrm{MeV}, 4440.3 \pm 1.3_{-4.7}^{+4.1} \mathrm{MeV}, 4457.3 \pm 0.6_{-1.7}^{+4.1} \mathrm{MeV}$ and corresponding decay widths $9.8 \pm 2.7_{-4.5}^{+3.7} \mathrm{MeV}, 20.6 \pm 4.9_{-10.1}^{+8.7} \mathrm{MeV}, 6.4 \pm 2.0_{-1.9}^{+5.7} \mathrm{MeV}$, respectively [1]. The state $P_{c}(4450)^{+}$in the 2015 data [2], is now replaced by two narrow states, $P_{c}(4440)^{+}$and $P_{c}(4457)^{+}$. In addition, a third narrow peak, $P_{c}(4312)^{+}$, having the mass $M=\left(4311.9 \pm 0.7_{-0.6}^{+6.8}\right) \mathrm{MeV}$, is also observed. The spin-parity, $J^{P}$, assignments of the three narrow states, which are crucial to decipher the underlying dynamics of the pentaquark states, are not yet determined. The broad peak $P_{c}(4380)^{+}$from the earlier data [2] is neither confirmed nor refuted, as the current LHCb analysis is not sensitive to broad resonances.

The constituents of the hidden-charm pentaquarks in the compact diquark model are $[c q]_{\overline{3}}, \bar{c}_{\overline{3}}$, and $\left[q^{\prime} q^{\prime \prime}\right]$, which make up a color singlet. However, it is still a three-body problem to solve and there are several dynamical possibilities to model their interconnections [3-6]. In [5, 6], we worked out the intuitive picture in which the heavier components form a nucleus and the lighter one is in an orbit around this nucleus, as it is energetically easier to excite light degrees of freedom. Spin-parity quantum numbers are fixed due to heavy-quark symmetry constraints. So, we keep the light diquark as emerging intact in $b$-baryon decays, and put it in the orbit for the $P$-wave states, with the heavier components, carrying a charm quark or charm antiquark, acting as a nucleus of (an almost) static color source.

Mass estimates are worked out in an effective Hamiltonian approach, which apart from constituent quark and diquark masses, includes dominant spin-spin, spin-orbit, orbital, and tensor interactions [5, 6]. Our mass predictions for the unflavored $(S=0)$, singly- $(S=-1)$ and doublystrange $(S=-2)$ hidden-charm pentaquarks with a light spinless diquark $\left(S_{l d}=0\right)$ are collected in Table 1. These states can be observed in weak decays of the bottom baryons. Masses of the states which are candidates for the already observed unflavored pentaquarks by the LHCb Collaboration are put into boxes in the table. Note that the singly- and doubly-strange pentaquarks mentioned have a strange light diquark $(S=-1)$, i. e. having the structures $\left(\bar{c}_{\overline{3}}[c q]_{\overline{3}}\left[s q^{\prime}\right]_{\overline{3}}\right)$ and $\left(\bar{c}_{\overline{3}}[c s]_{3}[s q]_{\overline{3}}\right)$, where $q^{(\prime)}$ is $u$ - or $d$-quark. Complete mass spectrum of the ground and orbitally excited hidden-charm pentaquarks can be found in [6].

We briefly comment on the recent evidence of a hidden-charm resonance, $P_{c s}(4459)$, with a strangeness $S=-1$. It was found in the $J / \psi \Lambda$ invariant mass spectrum in the $\Xi_{b}^{-} \rightarrow J / \psi \Lambda K^{-}$ decay with a statistical significance of $3.1 \sigma$ [7]. The Breit-Wigner mass and decay width of this state are measured to be $M_{P_{c s}(4459)}=4458.8 \pm 2.9_{-1.1}^{+4.7} \mathrm{MeV}$ and $\Gamma_{P_{c s}(4459)}=17.3 \pm 6.5_{-5.7}^{+8.0} \mathrm{MeV}$ but its spin and parity are not yet determined. Several assignments have been suggested for the $P_{c s}(4459)$-pentaquark and we also present a possible assignments based on the diquark-diquarkantiquark model of pentaquarks [6]. As shown in Table 1 (see the second column with mass estimates), the mass of the $P_{c s}(4459)$-resonance is well correlated with the $J^{P}=1 / 2^{-}$state having the mass $M=4433 \pm 26 \mathrm{MeV}$.

Experimental determination of the spins and parities of the $\mathrm{LHCb}$ pentaquarks will shed light on their underlying structures and allow us to discriminate among the suggested models for their 
Table 1: Masses of the unflavored $(S=0)$, singly-strange $(S=-1)$ and doubly-strange $(S=-2)$ hiddencharm pentaquarks (in $\mathrm{MeV}$ ) with a light diquark in the spin-0 state.

\begin{tabular}{|ccccc|}
\hline & $J^{P}$ & $S=0$ & $S=-1$ & $S=-1$ \\
\hline \multirow{4}{*}{$L=0$} & $1 / 2^{-}$ & $3830 \pm 34$ & $4112 \pm 32$ & $4243 \pm 32$ \\
& & $4150 \pm 29$ & $4433 \pm 26$ & $4575 \pm 26$ \\
& $3 / 2^{-}$ & $4240 \pm 29$ & $4523 \pm 26$ & $4644 \pm 26$ \\
& $1 / 2^{+}$ & $4030 \pm 39$ & $4312 \pm 37$ & $4443 \pm 38$ \\
& & $4351 \pm 35$ & $4633 \pm 33$ & $4775 \pm 33$ \\
\multirow{4}{*}{$L=1$} & $3 / 2^{+}$ & $4040 \pm 39$ & $4323 \pm 37$ & $4454 \pm 38$ \\
& & $4361 \pm 35$ & $4643 \pm 33$ & $4785 \pm 33$ \\
& & $4440 \pm 35$ & $4723 \pm 33$ & $4844 \pm 33$ \\
& $5 / 2^{+}$ & $4457 \pm 35$ & $4740 \pm 33$ & $4861 \pm 33$ \\
\hline
\end{tabular}

description.

\section{Acknowledgments}

A. P. acknowledges financial support by the Russian Foundation for Basic Research and National Natural Science Foundation of China according to the joint research project (No. 19-52-53041).

\section{References}

[1] LHCв collaboration, Observation of a narrow pentaquark state, $P_{c}(4312)^{+}$, and of two-peak structure of the $P_{c}(4450)^{+}$, Phys. Rev. Lett. 122 (2019) 222001 [1904.03947].

[2] LHCв collaboration, Observation of $J / \psi p$ Resonances Consistent with Pentaquark States in $\Lambda_{b}^{0} \rightarrow J / \psi K^{-} p$ Decays, Phys. Rev. Lett. 115 (2015) 072001 [1507.03414].

[3] R. F. Lebed, The Pentaquark Candidates in the Dynamical Diquark Picture, Phys. Lett. B 749 (2015) 454 [1507.05867].

[4] A. Ali, I. Ahmed, M. J. Aslam and A. Rehman, Heavy quark symmetry and weak decays of the b-baryons in pentaquarks with a ci component, Phys. Rev. D 94 (2016) 054001 [1607.00987].

[5] A. Ali and A. Y. Parkhomenko, Interpretation of the narrow $J / \psi p$ Peaks in $\Lambda_{b} \rightarrow J / \psi p K^{-}$ decay in the compact diquark model, Phys. Lett. B 793 (2019) 365 [1904.00446].

[6] A. Ali, I. Ahmed, M. J. Aslam, A. Y. Parkhomenko and A. Rehman, Mass spectrum of the hidden-charm pentaquarks in the compact diquark model, JHEP 10 (2019) 256 [1907.06507].

[7] LHCв collaboration, M. Wang, Recent results on exotic hadrons at LHC, 2020. 